Radioprotection, DunoD, 1978

Vol. $13, \mathrm{n}^{\circ} 1$, pages $11-21$

\title{
Effets biologiques des infrarouges et des ultraviolets
}

\author{
S. DESPRES (*) \\ (Manuscrit reçu le 3 décembre 1976) \\ RÉSUMÉ
}

Rayonnements électromagnétiques non ionisants, les infrarouges et les ultraviolets font partie de l'environnement normal de tout individu.

Leurs effets bénéfiques (apport calorique des infrarouges, synthèse de vitamine D favorisée par les ultraviolets...) qui justifient de nombreuses applications industrielles, domestiques ou médicales, s'accompagnent parfois d'effets nocifs, limités à la peau et aux yeux.

Ces manifestations pathologiques, précoces - comparables à des brûlures plus ou moins sévères - ou tardives - cancers cutanés, cataractes -, peuvent être évitées par le port de vêtements et de verres protecteurs ainsi que par le respect des normes d'expositions proposées par l'O.M.S.

\begin{abstract}
Among non-ionizing electromagnetic radiations, infrared and ultraviolet radiations are part of our normal environment. Their benefic effects (calory supply by infrared radiation, synthesis of vitamin D enhanced by ultraviolet radiation,...) justify many industrial, household or medical applications, yet there are sometimes noxious effects limited to the skin and eyes. The early pathological troubles, much like more or less severe burns, as well as late injuries-skin cancers, cataracts - can be avoided by protective clothes and glasses and by complying with the exposure standards set forth by W.H.O.
\end{abstract}

\section{RAPPEL \\ DE QUELQUES PROPRIÉTÉS PHYSIQUES DES RAYONNEMENTS INFRAROUGES ET ULTRAVIOLETS}

Possédant la même nature corpusculaire et ondulatoire que la lumière visible qu'ils encadrent dans le spectre des radiations électromagnétiques (tableau I), les rayonnements infrarouges et ultraviolets sont constitués de photons décrivant, dans le vide, des trajectoires rectilignes, à une vitesse voisine de $300000 \mathrm{~km} / \mathrm{s}$.

(*) Centre de Recherche de Médecine aéronautique, Laboratoire central de Biologie aéronautique, 5 bis, avenue de la Porte de Sèvres, 75731 Paris Cedex 15.

RADIOPROTECTION, VOL. $13-\mathrm{N}^{\circ} 1$ 
TABLEAU I

RADIATIONS ÉLECTROMAGNÉTIQUES

\begin{tabular}{|c|c|c|c|}
\hline \multicolumn{2}{|c|}{ Longueur d'onde } & \multirow{2}{*}{$\begin{array}{l}\text { Énergie photon } \\
\qquad(\mathrm{eV})\end{array}$} & \multirow[b]{2}{*}{ Radiations } \\
\hline (m) & $\begin{array}{l}\text { Autres } \\
\text { unités }\end{array}$ & & \\
\hline $10^{-14}$ & & $1,24.10^{5}$ & \multirow{4}{*}{ Rayons cosmique } \\
\hline $10^{-13}$ & 1 u. $x$. & $1,24.10^{7}$ & \\
\hline $10^{-11}$ & & $1,24.10^{5}$ & \\
\hline $10^{-10}$ & $1 \mathrm{~A}$ & $1,24.10^{4}$ & \\
\hline $10^{-8}$ & & $1,24.10^{2}$ & \multirow{2}{*}{$\begin{array}{l}\text { Ultraviolet } \\
\text { visible }\end{array}$} \\
\hline $10^{-6}$ & $1 \mu$ & 1,24 & \\
\hline $10^{-3}$ & $1 \mathrm{~mm}$ & $1,24 \cdot 10^{-3}$ & \multirow{2}{*}{ Ondes hertziennes } \\
\hline $10^{3}$ & $1 \mathrm{~km}$ & $1,24 \cdot 10^{-9}$ & \\
\hline
\end{tabular}

\begin{tabular}{|c|c|c|}
\hline$(\AA)$ & \multicolumn{2}{|c|}{ Ultraviolets (U.V.) } \\
\hline 136 & & \\
\hline 500 & Millikan & extrême \\
\hline 1200 & Lyman & (C) \\
\hline 1850 & Schumann & \\
\hline 2800 & Lointain & \\
\hline 3150 & Moyen & (B) \\
\hline 4000 & Proche & (A) \\
\hline
\end{tabular}

\begin{tabular}{|c|l|}
\hline$\mu \mathrm{m}$ & \multicolumn{1}{|c|}{ Infrarouge } \\
\hline 0,78 & \\
1,4 & Proche (A) \\
3 & Moyen (B) \\
1000 & Lointain (C) \\
\hline
\end{tabular}

L'énergie de ces photons est déterminée par la longueur d'onde de l'onde vibratoire qui leur est associée, selon la relation classique $W=h \mathrm{v}$, $h$ étant la constante de PLANCK.

Reçus par l'cil, la peau, ou tout autre récepteur quí les absorbe, ils sont responsables d'un échauffement, d'un déplacement d'électrons, d'une réaction chimique ou d'une émission secondaire de lumière:

La puissance transportée par un faisceau lumineux est appelée son flux d'énergie. Elle décroît par diffusion et par absorption lorsque le faisceau traverse les milieux matériels, se réfléchissant et se réfractant à leurs interfaces' successives. Dans un milieu homogène, l'extinction progressive du faisceau suit une loi exponentielle.

Les limites du spectre visible sont mal définies. Dans le rouge extrême, des lumières de longueur d'onde dépassant $0,85 \mu \mathrm{m}$ sont perceptibles par certains yeux, pourvu que la puissance qu'elles transportent soit suffisante 
(d'où un danger de brûlures graves). On peut admettre, par convention, que le domaine de l'infrarouge débute à $0,78 \mu \mathrm{m}$, valeur pour laquelle l'efficacité lumineuse est réduite à moins de $2 \cdot 10^{-5}$ de ce qu'elle est pour la lumière d'efficacité maximale $(0,555 \mu \mathrm{m})$. On peut, d'autre part, le limiter à la longueur d'onde supérieure $1 \mathrm{~mm}$, le raccord entre les ondes hertziennes et l'infrarouge lointain ayant été établi vers 1923 par Nichols et TeAR.

Ainsi limitées, on distingue, parmi les radiations infrarouges :

- l'infrarouge proche (ou A), compris entre 0,78 et $1,4 \mu \mathrm{m}$;

- l'infrarouge moyen (ou B), compris entre 1,4 et $3 \mu \mathrm{m}$;

- l'infrarouge lointain (ou C), compris entre 3 et $1000 \mu \mathrm{m}$.

Ces limites correspondent, en pratique, à des changements de moyens d'étude ou de production, les sources les plus utilisées étant, d'une part, l'arc au mercure à enveloppe de quartz, d'autre part, des corps incandescents comme le soleil, l'arc électrique ou le bec AUER.

Vers les courtes longueurs d'onde, les limites du spectre visible sont aussi imprécises. Ainsi, des lumières de longueur d'onde inférieures à $0,35 \mu \mathrm{m}$ peuvent être perçues si elles sont suffisamment intęnses. On convient souvent d'adopter, pour limites de l'ultraviolet, les valeurs de $0,40 \mu \mathrm{m}$ (pour lesquelles la sensibilité de l'cil moyen est, à puissance reçue, égale ou voisine de $4 \cdot 10^{-4}$ fois sa valeur maximale) et de $0,30136 \mu \mathrm{m}(136 \AA)$.

On dénomme :

- ultraviolet proche (A), le domaine des longueurs d'onde comprises entre 4000 et $3150 \AA$;

- ultraviolet moyen (B), celui compris entre 3150 et $2800 \AA$;

— ultraviolet lointain et extrême (C), celui compris entre 2800 et $136 \AA$

On appelle, parfois, du nom des savants qui les ont étudiés :

- ultraviolet de SchumanN, le domaine qui va de 1850 à $1200 \AA$;

- ultraviolet de LYMAN, celui qui va de 1200 à $500 \AA$;

- ultraviolet de Millikan, celui qui va de 500 à $136 \AA$.

Ces deux derniers ne peuvent être étudiés que dans le vide, l'air étant trop absorbant, et à l'aide de réseaux.

Le spectre solaire s'arrête pratiquement à la limite du proche ultraviolet. Celui de l'arc au charbon s'étend au début de l'ultraviolet lointain. Dans l'extrême ultraviolet, seules les étincelles dans un gaz raréfié peuvent être utilisées.

\section{EFFETS BIOLOGIQUES DES RAYONNEMENTS INFRAROUGES}

Absorbés, les infrarouges libèrent leur énergie essentiellement sous forme de chaleur et ce sont leurs effets thermiques qui ont permis, en 1801, à W. HerSCHEL, de les découvrir. C'est également cette action calorigène qui est recherchée dans l'utilisation industrielle, domestique ou médicale des infrarouges.

voL. $13-\mathrm{N}^{\circ} 1$ 
Les effets biologiques de ces rayonnements seront donc presque exclu sivement d'origine thermique, tout au moins dans leurs manifestations pathologiques, et ils se limiteront à la surface de l'organisme et aux tissus sous-cutanés. En effet, en raison de leur longueur d'onde, leur pénétration en profondeur n'excède pas $10 \mathrm{~mm}$.

Les personnes particulièrement exposées aux actions nocives des infrarouges sont les ouvriers des " industries du feu " (sidérurgie, soudure, verre et poterie, cuisson des peintures et revêtements émaillés) et, à un degré moindre, les utilisateurs d'appareils de chauffage (panneaux radiants au gaz ou émetteurs électriques) dont le principe est justement l'émission de ce type de rayonnement.

C'est ainsi que des employés travaillant dans des locaux chauffés par cette méthode se sont plaints de céphalées, de soif intense et de sécheresse de la peau.

\section{1) AU Niveau de LA PEAU}

L'action des infrarouges est habituellement immédiate. Elle est conditionnée par leur longueur' d'onde dont dépend l'absorption par le revêtement cutané. Ainsi, la transparence cutanée est totale pour les infrarouges de longueur d'onde inférieure à $1,5 \mu \mathrm{m}$, alors qu'entre 1,5 et $5 \mu \mathrm{m}$, il existe une forte absorption par l'épiderme et le derme. Pour les longueurs d'onde supérieures à $5 \mu \mathrm{m}$, la couche cornée de la peau arrête la totalité des rayonnements.

Par ailleurs, une peau rugueuse est moins absorbante qu'une peau lisse. Sa pigmentation, son épaisseur, sa vascularisation, son degré d'hydratation et sa température, sont autant de facteurs capables de modifier le pouvoir absorbant de la peau (la transmission par l'épiderme est diminuée de 10 à $30 \mathrm{p}$. cent lorsque celui-ci est humide, d'où le rôle protecteur de la sueur).

En règle générale, les effets demeurent superficiels pour les longueurs d'onde supérieures à $1,5 \mu \mathrm{m}$, alors qu'en-dessous de cette valeur, ils peuvent être plus profonds. Ces effets sont essentiellement :

- les brûlures,

- la dermite réticulaire.

En effet, les conséquences de l'hypersudation (surinfections microbiennes ou mycosiques), créée par l'exposition aux infrarouges, ne sont pas plus spécifiques que les coups de chaleur dont ils peuvent également être responsables.

\section{Les brûlures}

Elles sont, en général, superficielles. Succédant à un érythème et à un œdème du derme, elles se manifestent par des phlyctènes ou, pour des expositions plus importantes, par de véritables nécroses cutanées qui, lorsqu'elles se répètent fréquemment, pourraient favoriser l'apparition d'épithéliomas spinocellulaires.

Ces brûlures s'accompagnent rarement de choc et ne possèdent aucun caractère histologique particulier. 


\section{La dermite réticulaire}

Cette affection est favorisće par les troubles circulatoires, endocriniens, hépatiques, neuro-végétatifs, ainsi que par une baisse de l'état général ou un terrain allergique.

Elle débute par la formation d'un réseau violacé à larges mailles, accompagnant une vaso-dilatation intense et durable. Puis, les lésions deviennent bistres ou brun foncé, se continuant insensiblement avec la peau saine. Parfois, s'y associent sensation de brûlure, prurit et desquamation.

\section{2) L'GIL}

L'œil est l'autre organe-cible. En effet, hypermétrope pour les infrarouges, il est constitué de milieux très absorbants pour ce type de rayonnement. On admet que $30 \mathrm{p}$. cent environ des rayons infrarouges sont retenus dans le cristallin, cette proportion augmentant encore avec l'âge.

Pour les longueurs d'onde supérieures à $13 \mu \mathrm{m}$, les infrarouges sont inoffensifs car non pénétrants. Toutefois, focalisés sur l'œil, ils peuvent être la cause de conjonctivites.

Au-dessous de $13 \mu \mathrm{m}$ et pour un flux d'énergie important, les différentes structures de l'œil peuvent être lésées, entraînant atrophie de l'iris, altération du sphincter papillaire et de l'humeur aqueuse, anneaux de dépigmentation, lésions du cristallin, de la rétine et de la choroïde.

Pour des dọses modérées, on observe parfois une hyperhémie irienne.

La cataracte "du feu », dont sont victimes les verriers, a été contestée. Elle survient avec une fréquence variant entre 5 et 29 p. cent selon les auteurs, après 10 à 15 ans d'exposition intense journalière. Il s'agit d'une opacification intéressant initialement la face postérieure du cristallin, puis la totalité, et s'accompagnant parfois de craquelures radiées sur la capsule antérieure et de décollement de la zonule de ZINN. En fait, si elle est comparable à la cataracte sénile dans l'apparition de laquelle on a pu faire intervenir les infrarouges solaires, elle en diffère par l'âge plus précoce à laquelle elle survient.

La rétine peut être lésée dans certaines circonstances. Cela s'est vu notamment lors d'éclipses solaires où certaines personnes, ignorant un rayonnement infrarouge qu'elles ne voyaient pas, ont fixé le soleil pendant un temps trop long. Un nombre appréciable d'entre elles ont présenté un scotome définitif affectant leur vision centrale. En effet, leur cristallin, se comportant comme toute lentille convergente, avait focalisé les rayons infrarouges sur leur rétine, créant ainsi une coagulation thermique au niveau de la fovéa.

\section{LASERS A INFraROUGES (MASERS)}

Aux effets thermiques des infrarouges que nous venons de voir, s'ajoutent des effets mécaniques plus spécifiques (rupture traumatique des structures cellulaires et tissulaires).

voL. $13-\mathrm{N}^{0} 1$ 


\section{EFFETS BIOLOGIQUES DES RAYONNEMENTS ULTRAVIOLETS}

L'énergie des photons ultraviolets est de quelques électrons-volts (eV), c'est-à-dire supérieure (mais du même ordre de grandeur) à celle des liaisons intramoléculaires. On conçoit done aisément que lors de leur absorption par les molécules, celles-ci puissent subir des ruptures de leurs liaisons internes, des dissociations et des recombinaisons (dimérisations, polymérisations).

Cette connaissance de l'action des rayons ultraviolets à l'échelon moléculaire permet de mieux comprendre leurs effets biologiques sur la cellule et sur l'organisme entier.

\section{1) EFFets des ultraviolets SUR Les protéines et Les ACides NUClélQues}

Les pics d'absorption se situent à $2600 \AA$ pour l'ADN et à $2850 \AA$ pour l'albumine sérique, c'est-à-dire à la limite entre l'ultraviolet moyen et le lointain. Les conséquences de l'absorption des photons ultraviolets par ces grosses molécules ont été plus spécialement étudiées sur l'ADN des bactéries où on a pu mettre en évidence la formation de dimères entre bases pyrimidiques adjacentes situées le long d'une même chaîne de la molécule. Parmi ces dimères, les plus fréquents sont les dimères de thymine. Leur nombre est proportionnel à la dose reçue. In vivo, il se produit habituellement une réparation, favorisée par exposition à la lumière (photorestauration). Sous l'action d'endo puis d'exonucléases, les zones contenant les dimères sont excisées et remplacées par réplication de la chaîne opposée intacte (intervention d'une ADN polymérase). Une ADN ligase réalise ensuite la soudure des segments nouvellement synthétisés à la chaine polynucléotidique.

Chez les mammifères, un processus analogue interviendrait comportant, en outre, des phénomènes de recombinaison.

Une dose excessive conduit à l'accumulation de lésions réversibles mais dépassant les capacités de réparation des cellules. La synthèse de l'ADN est interrompue et la mort cellulaire survient avant la mitose.

L'expression de la survie cellulaire en fonction de la dose est une courbe exponentielle décroissante, comme avec les rayons $\mathrm{X}$, mais ici, la dose nécessaire pour tuer une cellule est 5000 fois plus forte et la période la plus sensible du cycle cellulaire est la phase " $\mathrm{S}$ ". La traduction microscopique des lésions de l'ADN apparaît au niveau des noyaux cellulaires dont la chromatine et les chromosones s'épaississent; le nucléole paraît gonfler et finalement se produit une picnose et la mort de la cellule.

C'est cet effet létal qui est recherché dans l'utilisation bactéricide et germicide des ultraviolets (stérilisation de l'atmosphère et des plans de travail en milieu hospitalier ou dans certains lieux nécessitant l'absence de germes. 


\section{2) EFFETS DES ULTRAVIOLETS SUR L'ORganisme HUMAIN}

Comme pour les infrarouges, le pouvoir de pénétration des ultraviolets est très limité. Ainsi, la couche cornée superficielle de la peau arrête les ultraviolets dont la longueur d'onde est inférieure à $2800 \AA$. Ceci explique que les effets directs de ces rayonnements n'intéressent guère que la peau (derme et surtout épiderme) et des organes comme les yeux.

Alors que les infrarouges ont un effet immédiat, les ultraviolets ont un effet généralement différé, avec un temps de latence parfois très long. Ainsi en est-il des cancers cutanés survenant longtemps après une exposition chronique aux ultraviolets solaires.

\subsection{Effets sur la peau}

Si on excepte la formation de vitamine D, la plupart de ces effets sont nocifs pour l'individu et apparaissent après des expositions excessives ou répétées, au rayonnement solaire, par exemple. Ils peuvent être directs ou indirects, nécessitant la présence de facteurs endogènes ou exogènes, et apparaître de façon immédiate ou différée.

- Les effets directs immédiats se manifestent dans le classique coup de soleil et vont du simple érythème cutané, qui apparaît 6 à 12 heures après l'exposition initiale et atteint son maximum autour de la $24^{e}$ heure, à des réponses plus intenses où existent douleur, adème, formation de phlyctènes. Dans ce dernier cas, les signes cutanés s'accompagnent fréquemment des symptômes généraux de toxiréaction (fièvre, frissons, nausées, délire et prostration). Des études histologiques de biopsies cutanées ont montré la -nature exacte des lésions. Initialement, il se produit un dépôt de glycógène dans les cellules basales de l'épiderme et une vasodilatation capillaire par action d'un médiateur chimique, grosse molécule dont le temps de diffusion expliquerait l'apparition différée de l'érythème. Les altérations cellulaires (picnose des noyaux, densification cytoplasmique), n'apparaissent pas avant 24 heures et sont localisées au corps muqueux de Malpighi. Nous avons vu que ce retard tenait aux altérations de l'ADN qui ne se révèlent qu'au moment de la mitose.

L'évolution des lésions se fait vers la desquamation (la peau « pèle "), suivie d'une intense activité mitotique aboutissant à la restauration de la couche de l'épiderme détruite et même à son épaississement. Parallèlement, la pigmentation cutanée augmente, parfois d'une manière non homogène et inesthétique lorsque la réaction a été trop sévère.

- Beaucoup plus sérieuses sont les manifestations différées. La dose totale requise pour les voir apparaitre n'est pas connue, mais on sait que toute exposition prolongée aux ultraviolets crée des lésions cutanées irréversibles dont l'aboutissement est la « peau sénile ". Celle-ci parait plus épaisse, plissée et écailleuse, parsemée de nombreuses taches pigmentées. Ces changements s'observent surtout au niveau de la face et du cou, ainsi que sur le dos des mains, zones habituellement exposées au soleil. Leur support histologique est une dégénérescence basophile des faisceaux de collagène et une fragmentation des fibres élastiques (élastose solaire ou

voL. $13-\mathrm{N}^{\circ} 1$ 
sénile). Ils précèdent fréquemment des lésions précancéreuses - kératoses actiniques ou séniles - et de vrais cancers cutanés : épithéliomas spinocellulaires récidivant sur place ou métastasant à distance. Les carcinomes basocellulaires et les mélanomes, bénins ou malins, surviennent egalement avec une plus grande fréquence sur les peaux exposées fréquemment aux rayonnements ultraviolets.

Il est théoriquement possible de recevoir une dose létale de radiations ultraviolettes puisqu'il n'existe pas de détecteur cutané et que l'érythème n'y apparaît que tardivement. En réalité, l'émission d'ultraviolets s'accompagne habituellement d'autres rayonnements, visibles ou infrarouges, immédiatement perceptibles par un individu à l'état de veille.

- Les réactions indirectes ou de photosensibilité sont multiples (une quarantaine au moins ont été décrites). Il peut s'agir d'un simple érythème sans œdème, d'un urticaire, de papules, de vésicules, ou même d'eczéma.

Les régions protégées par les cheveux, les plis cutanés et les vêtements, ne sont pas atteintes.

- La photosensibilité peut être d'origine endogène :

C'est le cas des porphyries acquises ou congénitales, pour lesquelles les expositions aux ultraviolets mais aussi à la lumière visible, sont responsables de lésions cutanées vésiculo-bulleuses, urticariennes, ou eczématiformes, suivies parfois de modifications de la pigmentation.

De même la pellagre et la maladie de HARTNuP pourraient être dues, au moins en partie, à la circulation de substances photosensibilisatrices.

Dans le Xeroderma pigmentosum, la cause de la photosensibilité serait un défaut génétique du système de réparation des lésions de l'ADN, induites par l'irradiation ultraviolette. Notons que, dans cette affection, les lésions précancéreuses et cancéreuses surviennent avec une plus grande fréquence.

Certaines maladies dermatologiques sont aggravées par l'exposition aux ultraviolets. Ainsi, en est-il de l'herpès, de la varicelle, de la lymphogranulomatose vénérienne, dont l'étiologie est connue, mais également d'affections comme le psoriasis, le lichen plan, la kératose folliculaire, le pityriasis, le pemphigus érythémateux, l'érytème polymorphe et certains eczémas. Le terme de phénomène de KOEBNER est parfois donné à ce type de manifestations pathologiques succédant à une exposition aux ultraviolets généralement d'origine solaire.

Une plus grande sensibilité cutanée aux ultraviolets peut aussi être due à un défaut de pigmentation de la peau tel qu'on l'observe dans l'albinisme, le vitiligo, l'hypopituitarisme, l'hypogonadisme et la phénylcétonurie. Le lupus érythémateux, maladie auto-immune du collagène, est considérablement aggravé par l'exposition aux ultraviolets auxquels sont particulièrement sensibles les sujets qui en sont atteints.

Les photosensibilités d'origine exogène sont souvent en rapport avec la prise de médicaments, et peuvent se produire sous forme d'une photo-allergie 
ou d'une phototoxicité. Le rayonnement responsable appartient à l'ultraviolet proche.

Les réactions phototoxiques, exceptionnellement observées chez les sujets de race noire, surviennent après usage interne ou externe d'antibiotiques (Tétracycline), d'antibactériens (sulfamides), d'antimycosiques (Griseofulvine), de phénothiazines (sédatifs, etc.), de chlorothiazides (diurétiques), de certains hypoglycémiants (sulfonylurées). Elles sont immédiates, consécutives à des expositions à un rayonnement ultraviolet intense et se traduisent par érythème, œdème, et phlyctènes. L'érythème peut persister plusieurs semaines et s'accompagner d'irrégularités dans la pigmentation.

Les agents cosmétiques provoquant des réactions phototoxiques ont souvent une action différée. C'est le cas des furocoumarines responsables de la phytophotodermatite qui apparaît entre 12 et 24 heures après l'application du produit (œdème, vésicules, irrégularités de la pigmentation).

Contrairement aux précédentes, les réactions photo-allergiques n'épargnent aucune race et nécessitent une sensibilisation à une substance chimique (haptène) produite par action des ultraviolets. Les mécanismes d'immunité cellulaire retardée sont impliqués dans ce type de réaction dont les manifestations habituelles (démangeaisons, eczéma, hypersudation,...) apparaissent 24 à 48 heures après l'exposition conjuguée au rayonnement ultraviolet et à l'haptène sensibilisateur et se prolongent pendant une dizaine de jours. Lorsqu'il y a répétition de l'agression, la peau s'épaissit, se fissure et devient hyperpigmentée. De faibles doses d'exposition suffisent à induire les réactions photo-allergiques.

- Pigmentation de la peau et irradiation par les ultraviolets :

Après exposition aux ultraviolets naturels (rayonnement solaire) ou artificiels, la peau devient plus foncée (bronzage). Trois mécanismes interviennent dans ce phénomène :

1. Brunissement immédiat de là peau à partir du pigment mélanique présent et nécessitant la présence d'oxygène. Cet effet, variable d'un individu à l'autre, tend à diminuer après 45 ans.

2. Migration de la mélanine de la profondeur à la surface de l'épiderme. La localisation de la mélanine dans les couches superficielles, augmente son effet protecteur envers les ultraviolets, expliquant la résistance plus grande à ces radiations des sujets de race noire.

3. Accroissement de la quantité totale de mélanine présente dans la peau. L'activité en tyrosinase est en faveur d'une synthèse accrue de ce pigment.

On peut remarquer que le rôle protecteur de la mélanine à l'égard des ultraviolets ne s'étend pas au rayonnement infrarouge.

Synthèse de vitamine $D$

C'est le seul effet bénéfique certain des expositions aux ultraviolets. Il est dû à l'ouverture du cycle $\beta$ d'une molécule stérique grâce à l'apport énergétique des photons ultraviolets.

voL. $13-\mathrm{N}^{0} 1$ 


\subsection{Effets sur les yeux}

Nous ne parlerons pas de l'influence possible des ultraviolets sur la pigmentation de l'iris ni de leur perception éventuelle par la rétine, bien que ces sujets aient un grand intérêt sur le plan de la physiologie ou de l'anthropologie.

Fluorescent en lumière ultraviolette, le cristallin absorbe la majeure partie du rayonnement qui a franchi la cornée. Ainsi, peuvent survenir des cataractes de même que des lésions cornéennes. Quant aux conjonctivites, elles sont bien connues et fréquemment observées lorsque des personnes s'exposent aux ultraviolets sans se protéger les yeux par des verres absorbants.

Notons que certains établissent une relation entre la coloration de l'iris et la susceptibilité à développer des cancers cutanés après exposition aux ultraviolets.

\subsection{Autres effets sur l'organisme}

La pression sanguine est diminuée après exposition aux ultraviolets, de même que le taux de cholestérol sanguin et de tyrosine plasmatique (utilisée dans la synthèse de mélanine).

Par contre, l'hypothèse ancienne d'une action directe des ultraviolets sur l'encéphale destinée à expliquer les troubles ressentis par les Européens en zone tropicale, n'a aucun fondement sérieux.

D'autres effets, observés chez l'animal, n'ont guère été constatés chez l'homme. Ainsi, les ultraviolets favorisent l'agrégation des plaquettes en provoquant la libération de catécholamines par les mastocytes. Ils provoquent la relaxation des muscles lisses par modification de leur perméabilité cellulaire. Ce phénomène est surtout évident pour les artères. Chez le rat, les ultraviolets accélèrent l'apparition d'ulcères de contrainte.

D'une manière générale, les accidents dus aux ultraviolets sont la conséquence d'imprudence. Y sont exposés les utilisateurs d'émetteurs artificiels (soudure à l'arc, lampes ultraviolettes à usage thérapeutique ou esthétique). Le personnel effectuant des vols à haute altitude encourt également certains risques dus à la plus grande densité de ce rayonnement.

\section{MESURES DE PROTECTION ET LIMITES D'EXPOSITION}

\section{1) Infrarouges}

- La protection cutanée est assurée par le port de vêtements; la toile d'amiante est un excellent isolant. La ventilation des lieux de travail permet d'éviter les symptômes généraux dont le "coup de chaleur».

Les yeux seront protégés par des verres filtrants spéciaux (norme française NF B 36.007).

- Les normes définies par l'O.M.S. se rapportent aux lésions cornéennes. Les valeurs en sont :

- 7,6 J/ $\mathrm{cm}^{2}$ pour les longueurs d'onde comprises entre 0,88 et $1,1 \mu \mathrm{m}$;

$-2,8 \mathrm{~J} / \mathrm{cm}^{2}$ entre 1,2 et $1,7 \mu \mathrm{m}$. 


\section{2) Ultraviolets}

Envers les ultraviolets du rayonnement solaire, l'attitude à adopter va à l'encontre de la mode des bains de soleil à outrance.

Le port de verres protecteurs au pouvoir filtrant reconnu doit être la règle, notamment en altitude où le flux de photons ultraviolets est accru. Les vêtements, même légers, suffisent à absorber le rayonnement ultraviolet. En leur absence, on pourra utiliser des onguents et émulsions (benzophénones, etc.) qui ont un effet protecteur indéniable mais dont les applications doivent être fréquemment renouvelées.

- A l'échelle mondiale, un certain nombre de pays se soucient de l'intégrité de la couche d'ozone qui forme un écran protecteur indispensable à l'égard des ultraviolets solaires. Les réticences à l'égard des transports supersoniques et l'interdiction récente des produits en "bombe ", s'inscrivent dans ces préoccupations d'ordre écologique.

- Les recommandations de l'O.M.S. quant aux normes à adopter, sont : $0,5 \mu \mathrm{W} / \mathrm{cm}^{2}$ pour une exposition inférieure ou égale à 7 heures; $0,1 \mu \mathrm{W} / \mathrm{cm}^{2}$ pour une exposition continue de 24 , heures par jour.

Pour des expositions plus courtes à l'ultraviolet proche (3 200 à $4000 \AA$ ), la densité de puissance surfacique au niveau de la peau ou de l'cil non protégés ne devrait pas être supérieure à $1 \mathrm{~mW} / \mathrm{cm}^{2}$ pour des périodes de plus de 16 minutes. La densité d'énergie surfacique totale pour des expositions inférieures à 16 minutes ne devrait pas excéder $1 \mathrm{~J} / \mathrm{cm}^{2}$.

\section{CONCLUSION}

Les rayons infrarouges et ultraviolets, d'origine solaire ou artificielle, sont peu pénétrants et leur action est limitée à la peau et aux yeux. Le port de vêtements et de verres protecteurs suffisent à se protéger de leurs effets néfastes, que ceux-ci soient précoces (brûlures cutanées et rétiniennes, réactions de photosersibilisation), ou tardifs (lésions précancéreuses et cancéreuses, cataractes).

\section{BIBLIOGRAPHIE}

[1] Decaud J. Action pathologique des infrarouges. Thèse, Paris, 1955.

[2] Dunlaa C. E. Effects of radiation. In : Pathology, Anderson, W.A.D., Ed., St-Louis, C.V. Mosby, 1971, 1, 242-243.

[3] Daniess F. Man and radiant energy : solar radiation. In : Handbook of physiology, section 4, chap. 62, 969-987, Washington, American physiological society, 1964.

[4] Fleury P. et Mathieu J. P. Lumière. In : Physique générale et expérimentale. Paris, Eyrolles, 1961.

[5] JАMmet H. et Rodier J. Maladies causées par les rayonnements. Encyclopédie médicochirurgicale, Intoxications. Paris, 1975, 16510 A-10.

[6] STECK B. Photobiologische Wirkungen der Optischen Strahlung auf den Menschen. Lichttechnik, 1976, 28, 23-27.

[7] Tubiana M. Radiopathologie. In : La pathologie médicale. Paris, Flammarion, 1972.

[8] Wruts I. Sunlight and the skin. Jama, 1971, 217, 1088-1093.

VoL. $13-\mathrm{N}^{\bullet} 1$ 